\title{
Electron and hole storage in self-assembled InAs quantum dots
}

\author{
D. Heinrich*, J. Hoffmann, J.J. Finley, A. Zrenner, G. Böhm, G. Abstreiter \\ Walter Schottky Institut, Technische Universität München, Am Coulombwall, D-85748 Garching, Germany
}

\begin{abstract}
We present results on optically induced storage of electrons or holes in self-assembled InAs quantum dots (QDs). The measurements demonstrate that, following resonant photo-excitation of the QDs, excitons can be ionised selectively leaving either electrons or holes stored. The stored charge is sensed via resistivity changes in a remote $2 \mathrm{D}$ carrier system. The induced photo-effect is persistent over time scales of $>8 \mathrm{~h}$ at a temperature of $145 \mathrm{~K}$. A series of resonances are observed in the spectral characteristics of the photo-effect. The charging probability was derived from the analysis of the temporal behavior of this charge storage effect. (C) 2000 Elsevier Science B.V. All rights reserved.
\end{abstract}

PACS: $85.30 . \mathrm{Vw} ; 73.50 . \mathrm{Pz} ; 78.66$

Keywords: Quantum dots; Memory devices; Charge storage; Excitation spectroscopy

Self-assembled quantum dots (QDs) are particularly attractive since they appear to be defect free, have high areal densities and good optical quality [1,2]. However, large ensembles usually exhibit a strongly broadened absorption line shape which is associated with size fluctuations. Such uniformity fluctuations are undesirable for most device applications, but Muto et al. have suggested that these fluctuations may be advantageous for wavelength domain data storage [3]. In the case of QDs, information can be recorded as a small number of electrons or holes being stored within the deep confinement potential of the QDs. First efforts in this direction have been made [4] with data retention times up to $\sim 0.5 \mathrm{~ms}$ reported at room temperature.

\footnotetext{
* Corresponding author. Tel.: +49-89-289-12736; fax: +49-89-320-6620.

E-mail address: heinrich@wsi.tum.de (D. Heinrich)
}

Recently, several groups have investigated the effects of a self-assembled InAs QD layer on the lateral transport of a 2D electron gas in MODFET-type structures [5-8]. It was demonstrated that such devices are highly photo-sensitive, allowing for possible applications as QD optical memory elements combining in principle both ultra-dense storage capacities $\left(\sim 1 \mathrm{Tbit}^{-2}\right)$ [2] and very low switching energies.

In this paper, we report on spectrally resolved optical charging experiments performed on self-assembled InAs QDs storing either electrons or holes.

Our optically gated MODFET structures have the following layer sequence (described here for a p-channel device for hole storage): An $n$-type substrate and back contact followed by an undoped $240 \mathrm{~nm}$ GaAs spacer, an InAs QD layer deposited at $530^{\circ} \mathrm{C}$ and an AlAs/AlGaAs barrier. A GaInAs QW channel was then grown followed by a AlGaAs 


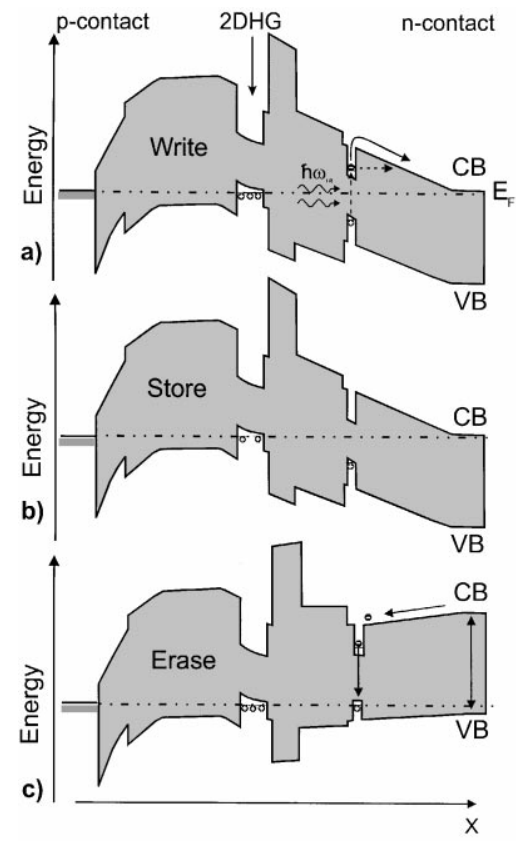

Fig. 1. Schematic operation principle of the storage device in the case of hole storage: (a) NIR illumination leads to charge storage in the InAs QDs; (b) The stored charge persists and depletes the 2D channel; (c) A reset pulse erases the stored charge in the QDs.

barrier containing two p-doping layers and a p-doped GaAs cap layer. A reference sample without QD layer was also fabricated. The wafers were processed into a Hall bar geometry with separate ohmic contacts established to the 2D channel and the back contact.

The n-channel device (for electron storage) is almost similar with $\mathrm{p}$ and $\mathrm{n}$ doping exchanged [6].

In Fig. 1, the fundamental operating principles of such a device is illustrated schematically. It shows the band diagram of a hole storage structure (the principles for electron storage are analogous).

By illuminating the sample with monochromatic near-infra-red (NIR) light, a subset of QDs is selectively excited (Fig. 1a). After excitation, the electrons (holes for the electron storage structure) are removed by the vertical electric field in the $\mathrm{p}-\mathrm{i}-\mathrm{n}$ junction, the holes (electrons) remain stored as a consequence of the AlGaAs barrier. The stored charge in the QD layer acts as gate-charge on the 2D channel, thus modifying the surface conductivity [5] (Fig. 1b). The magnitude of the resulting resistance change reflects the stored charge density. The saturation level of the channel re- sistance is expected to reflect all QDs which can be addressed by light of the selected optical excitation energy. All holes (electrons) stored may be removed by injecting electrons (holes) into the QD layer from the back contact (device reset) (Fig. 1c). The device then returns to its natural state, ready to perform further illumination-storage-reset cycles.

In order to investigate optically induced charge storage effects, the samples were illuminated with NIR light at a temperature of $T=145 \mathrm{~K}$. An increase of the channel resistance is observed after switching on the illumination (see Fig. 2a), saturating at a level denoted $R_{1}$. When the illumination is switched off, the resistance slightly recovers to $R_{2}$ (Fig. $2 \mathrm{a}$ ). The residual photo-effect, $\Delta R=R_{2}-R_{0}$, is persistent for $>8 \mathrm{~h}$ at $T=145 \mathrm{~K}$ and it can be observed up to $\sim 200 \mathrm{~K}$. This temperature dependence is caused by thermionic emission of stored charges out of the QDs which becomes more probable with increasing temperature.

The effect of exciting different dots within the ensemble was investigated for the hole storage structure by changing the excitation energy $\left(E_{\mathrm{ex}}\right)$ for each illumination-recovery-reset cycle.

A clear onset of the photo-resistance is observable at the ground state PL peak (Fig. 2b, full line). Towards higher energies resonant structures in the photo-resistance (Fig. 2b, circles) are observed. These structures are slightly shifted to higher energies as compared to the ground and excited states of the PL spectrum, respectively. The reference sample grown without dots shows no charging effect in the excitation energy range $0.96<E_{\text {ex }}<1.18 \mathrm{eV}$ (Fig. 2b, squares).

Performing this experiment without resetting the sample after illumination at each excitation energy $E_{\text {ex }}$ results in accumulative charging. The corresponding curve (Fig. 2b, triangles) does not reflect the resonances of the selective charging curve but it shows a slight step like behavior coinciding with the resonances observed when resetting the sample. The increase in resistance is larger because more charge is stored in total. Due to this accumulative excitation in a wider energy range all dots are addressed. In the energy regime of the excited states accumulative charging results in about twice the resistance chance as compared to selective charging (with reset).

In a simple model, the magnitude of the selective charging curve should be caused only by a small subset of QDs matching the excitation en- 

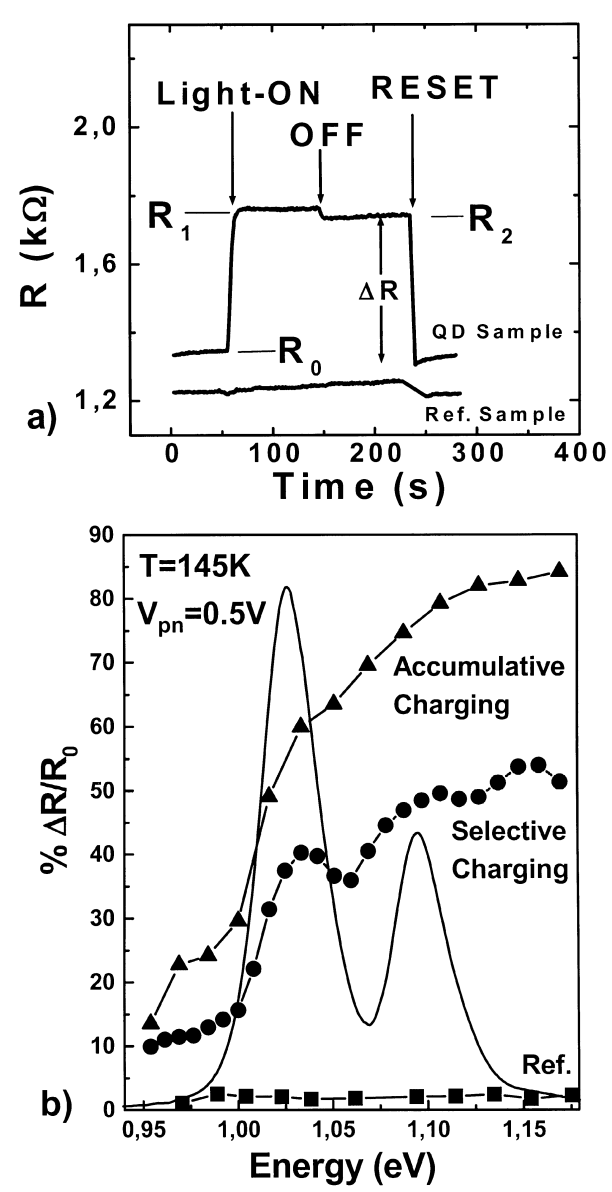

Fig. 2. (a) Typical illumination-recovery-reset cycle for a structure containing QDs (top) and reference structure (bottom). (b) Spectral dependence of photo-resistance measurement for $V_{\mathrm{pn}}=0.5 \mathrm{~V}$ performed on the hole storage structure. The full line shows the PL spectrum.

ergy, hence resulting in a small effect. The amount of charging observed in the experiment therefore indicates that additional dots can be charged by thermal feeding processes or phonon assisted absorption. Thermally assisted escape of stored charge from QDs to the wetting layer leads to thermal feeding of neighboring QDs. The escaped charge moves in the wetting layer and can be stored again in another QD probably with a lower ground state energy. Processes involving LO phonon assisted charging can address further sub-sets of QDs increasing the number of charged QDs for selective excitation.

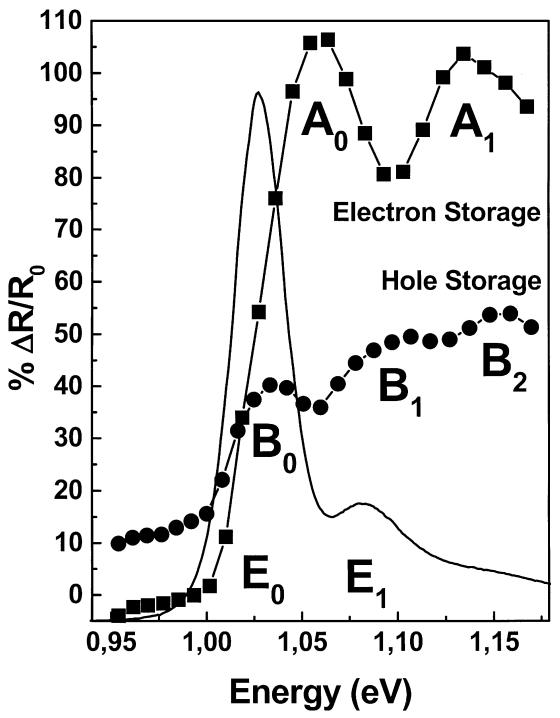

Fig. 3. Spectrally resolved resistance measurements of electron and hole storage samples. The magnitude of the resistance changes depends on the initial channel resistance and can be varied by reverse bias on the samples. The full line shows the PL spectrum of the electron storage sample.

Selective charging performed on electron storage structures also shows resonances in the photo-resistance (see Fig. 3, squares). PL measurements (full line) on the electron storage structure reveal a QD ground state emission at $E_{0}=1025 \pm 5 \mathrm{meV}$ (FWHM $\sim 30 \pm 5 \mathrm{meV})$, and excited state emission $\left(E_{1}, E_{2}\right)$ at $60 \pm 5$ and $120 \pm 5 \mathrm{meV}$ above the ground state emission. The spectral dependence of the photo-resistance effect $\left(\Delta R / R_{0}\right)$ shows resonances $\left(A_{0}\right.$ and $\left.A_{1}\right)$ with a separation of $75 \pm 5 \mathrm{meV}$. These resonances are shifted from $E_{0}$ by $33 \pm 5 \mathrm{meV}$ and $115 \pm 10 \mathrm{meV}$ for $A_{0}$ and $A_{1}$, respectively. This indicates that they do not simply correspond to the direct excitation of ground and excited QD states. However, the energy shift between $E_{0}$ and $A_{0}(33 \pm 5 \mathrm{meV})$ is very close to the LO phonon energy $\left(\hbar \omega_{\mathrm{LO}}\right)$ expected for InAs QDs in GaAs [9]. The participation of inelastic processes in optical QD excitation has been demonstrated on both large ensembles [1,9] and single dots [10], with a number of vibrational modes with energies ranging from $\sim 27$ to $37 \mathrm{meV}$. Previously, we already have tentatively suggested the participation of inelastic processes over the long time scales involved [6]. 


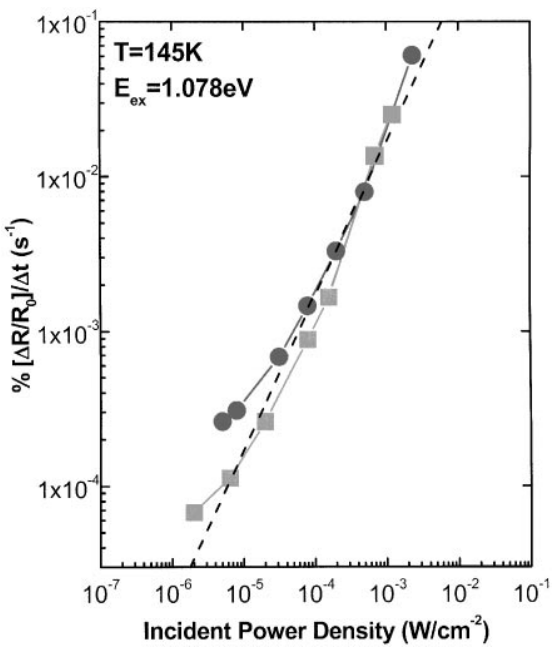

Fig. 4. Dependence of the rate of resistance change in the $2 \mathrm{D}$ channel on the incident optical power density.

Comparing the photo-resistance curves for hole storage (Fig. 3, circles) and electron storage (Fig. 3, squares), the resonances are both shifted toward higher energies with respect to the ground and excited states of the PL spectrum, respectively. There is a larger shift observed in the electron storage sample.

These resonances clearly indicate that the QDs have an enhanced capacity for charge storage at $A_{0}, A_{1}, A_{2}$ and $B_{0}, B_{1}, B_{2}$ (Fig. 3), reflected in the amount of charge stored but observable also in the charging rate. The speed at which the QDs are charged peaks also at these spectral positions. The phonon-assisted processes involved at these resonances are very effective for charging the InAs QDs.

The temporal dependence of the charge storage effect was investigated by changing the incident power density $\left(P_{\mathrm{ex}}\right)$ of the optical excitation. The charging rate as determined from the initial slope of the resistance changes increases with increasing power whereas the saturation level of the channel resistance remains independent of $P_{\mathrm{ex}}$. This indicates that a fixed number of QDs is charged, finally. Fig. 4 shows the charging rate of the hole (squares) and electron storage sample (circles). The dependence of the charging rate on the incident power density is approximately linear. A simple model was applied in order to understand these observations qualitatively. The density of uncharged QDs $\left(N_{0}\right)$ addressed by the optical ex- citation decreases with time while illuminated. This reflects the bleaching of the absorption in the charged QDs. Assuming that the QDs are non-interacting, $N_{0}$ decreases exponentially in time according to

$N_{0}=N_{\mathrm{QD}} \exp \left(-\frac{P_{\mathrm{ex}} \alpha}{E_{\mathrm{ex}} N_{\mathrm{QD}}} t\right)$

with $N_{\mathrm{QD}}$ being the total density of QDs addressed by the optical excitation and $\alpha$ the mean QD absorption strength reflecting the probability of charging a QD. Photo-generated electrons (holes for electron storage) escape faster out of the QDs than they recombine radiatively, leaving one charge stored. The density of charged QDs $\left(N_{+}\right)$is then $N_{+}=N_{\mathrm{QD}}-N_{0}$. These charged QDs reduce the carrier density of the 2D channel $\left(n_{2 \mathrm{D}}\right)$ by $n_{2 \mathrm{D}}=n_{2 \mathrm{D}}^{0}-f N_{+}$, where $f$ is an electrostatic leverage factor and $n_{2 \mathrm{D}}^{0}$ is the channel density before illumination. Approximately one hole in the 2D channel is depleted for each stored hole in the QD layer $\left(f=\left(1-d_{1} / d_{2}\right)^{-1}\right.$ with $d_{1}$ being the separation of the QD layer and $d_{2}$ of the back contact from the 2D channel).

The change in conductivity of the $2 \mathrm{D}$ channel $(\Delta \sigma)$ can now be written

$\frac{\mathrm{d} \sigma}{\mathrm{d} t}=e \frac{\mathrm{d} n_{2 \mathrm{D}}}{\mathrm{d} t}\left(\mu+n \frac{\partial \mu}{\partial n_{2 \mathrm{D}}}\right)$.

Providing the mobility of the $2 \mathrm{D}$ channel is not strongly perturbed by the charged QD layer this equation can be rewritten (using Eq. (1)) as

$$
\begin{aligned}
\frac{\mathrm{d} \sigma}{\mathrm{d} t} & =-\alpha\left(\frac{e f \mu}{\eta \omega}\right) P_{\mathrm{ex}} \exp \left(-\frac{\alpha P_{\mathrm{ex}}}{\eta \omega N_{\mathrm{QD}}} t\right) \\
& \approx-\alpha \frac{e f \mu}{\eta \omega} P_{\mathrm{ex}} .
\end{aligned}
$$

The approximation is valid for small times $(t \ll$ $\left.\eta \omega N_{\mathrm{QD}} / \alpha P_{\mathrm{ex}}\right)$ after the onset of illumination. It predicts a linear dependence of $\mathrm{d} \sigma / \mathrm{d} t$ on $P_{\text {ex }}$ as observed in Fig. 4 for electron and hole storage.

For the electron storage structure we obtain $\alpha=$ $3.5 \times 10^{-5}$. For the hole storage structure we find within the errorbars the same. Previously published results of absorption measurements of quantum dots [11] are comparable to this value.

In conclusion, we have demonstrated devices capable of hole or electron storage in InAs QDs resembling a quantum dot "optically gated" FET. Resonant optical excitation of the QDs results in photo-resistance 
resonances shifted to larger energies with respect to the PL energy. The excitation power dependence enables us to estimate the optical absorption strength for electrons and holes.

This work was supported financially by the Deutsche Forschungsgemeinschaft via SFB 348.

\section{References}

[1] M.J. Steer, D.J. Mowbray, W.R. Tribe, M.S. Skolnick, M.D. Sturge, Phys. Rev. B 54 (1996) 17738.

[2] D. Leonard, M. Krishnamurty, C.M. Reaves, S.P. Denbaars, P.M. Petroff, Appl. Phys. Lett. 63 (1993) 3203.

[3] S. Muto, Jpn. J. Appl. Phys. 34 (1995) L210.

[4] K. Imamura, Y. Sugiyama, Y. Nakata, S. Muto, N. Yokoyama, Jpn. J. Appl. Phys. 34 (1995) L1445.
[5] G. Yusa, H. Sakaki, Appl. Phys. Lett. 70 (1997) 345.

[6] J.J. Finley, M. Skalitz, M. Arzberger, A. Zrenner, G. Bohm, G. Abstreiter, Appl. Phys. Lett. 73 (1998) 2618.

[7] A.J. Shields, M.P. O’Sullivan, I. Farrer, D.A. Ritchie, K. Cooper, C.L. Foden, M. Pepper, Appl. Phys. Lett. 74 (1999) 735.

[8] E. Ribeiro, E. Miller, T. Heinzel, H. Auderset, K. Ensslin, Phys. Rev. B 58 (1998) 1506.

[9] R. Heitz, M. Grundmann, N.N. Ledentsov, L. Eckey, M. Veit, D. Bimberg, V.M. Ustinov, A.Yu. Egorov, A.E. Zhukov, P.S. Kop'ev, Zh.I. Alferov, Appl. Phys. Lett. 68 (1995) 361.

[10] A. Zrenner, M. Markmann, A. Paassen, A.L. Efros, M. Bichler, W. Wegscheider, G. Böhm, G. Abstreiter, Physica B 256-258 (1998) 300.

[11] R.J. Warburton, C.S. Dürr, K. Karrai, J.P. Kotthaus, G. Medeiros-Ribeiro, P.M. Petroff, Phys. Rev. Lett. 79 (1997) 5282. 